\title{
TRATAMENTO MEDICAMENTOSO DOS TRANSTORNOS PARAFÍLICOS
}

\author{
Arnaldo Barbieri Filho' \\ PARAPHILIC DISORDERS DRUG TREATMENT
}

\begin{abstract}
Resumo: O objetivo deste artigo foi apresentar as medicações utilizadas para o tratamento de Transtornos Parafílicos (TP), seus mecanismos de ação, efeitos colaterais e a importância das comorbidades presentes para a escolha da melhor droga para cada caso. Foi realizada revisão de literatura no Pubmed e Medline. Muitas medicações podem ser úteis: antidepressivos que agem no receptor serotoninérgico $5 \mathrm{HT} 2 \mathrm{a}$, especialmente em casos de depressão e doenças ansiosas; antipsicóticos, que são antidopaminérgicos, em psicoses; estabilizadores de humor em transtorno bipolar e impulsividade; naltrexona no alcoolismo; antiandrógenos de ação periférica ou central como os agonistas parciais do hormônio liberador de gonadotrofina hipotalâmico (agonistasLHRH), em casos mais severos. O melhor tratamento para cada indivíduo depende dos diagnósticos (sexual, psiquiátrico e médico geral). Tratar um indivíduo com doença de Wilson, psicose orgânica e transtorno exibicionista é diferente de tratar um indivíduo com o mesmo TP, hipotireoidismo e depressão. É também importante tentar preservar a função sexual da maneira mais saudável possível. Por outro lado, é fundamental proteger a sociedade dos agressores sexuais. Questões éticas como a possibilidade de toxicidade das medicações devem ser amplamente discutidas e documentadas. Destaca-se o fato de que o uso de antiandrógenos para castração química não é liberado no Brasil.
\end{abstract}

Palavras-chave: transtornos parafílicos; comorbidades; tratamento medicamentoso.

\begin{abstract}
The present paper aims to study the medications used to treat Paraphilic Disorders (PD), their mechanisms of action, side effects and the importance of comorbidities to choose the best drug for each case. A literature search was conducted at Pubmed and Medline. Many medications can be helpful: Antidepressants acting on 5HT2a serotonergic receptor, especially in depression and anxious diseases; Antipsychotics, that are antidopaminergic, in psychosis; Mood Stabilizers in Bipolar Disorder and impulsiveness; Naltrexone in alcoholism; peripheral or central acting Antiandrogens as the partial agonists of hypothalamic Luteinizing hormone-releasing hormone (LHRH agonists), in more severe cases. The best treatment for each individual depends on the diagnosis (Sexual, Psychiatric and General Medical). Treating an individual with Wilson's Disease, Organic Psychosis, and Exhibitionistic Disorder is different from treating an individual with Hypothyroidism, Depression and Exhibitionistic Disorder. It is also important to try to preserve sexual function in the healthiest way possible. On the other hand, it is fundamental to protect society from sexual offenders. Ethical issues as a possibility of the toxicity of medications should be widely discussed and documented.It is important to note that the use of antiandrogens for chemical castration is not allowed in Brazil.
\end{abstract}

Keywords: paraphilic disorders; comorbidities; drug treatment. 


\section{Introdução}

Quando o assunto é parafilia, o que deveria ser considerado doença e o que não deveria foi alvo de muitas polêmicas ao longo da história (KRAFFT-EBING, 1965; MONEY, 2003; DE BLOCK et al., 2013; KAPFHAMMER, 2015). O meio científico era influenciado por conceitos religiosos e pensamentos preconceituosos (WAKEFIELD, 2011).

Em 2013, a American Psychiatric Association (APA) publicou o Manual Diagnóstico e Estatístico de Transtornos Mentais (DSM-5) (APA, 2013), que também foi alvo de várias controvérsias (KRUEGER, 2010a; KRUEGER, 2010b; KRUEGER, 2011; FEDOROFF, 2011; WAKEFIELD, 2011; DE BLOCK et al., 2013; FEDOROFF et al., 2013). O DSM-5 distinguiu, então, parafilia de transtorno parafílico (TP). O termo parafilia representa qualquer interesse sexual intenso e persistente que não aquele voltado para estimulação genital ou para carícias preliminares com parceiros humanos capazes de consentir, que tenham fenótipo normal e maturidade física. Esse interesse intenso e persistente deve ocorrer por pelo menos seis meses (critério $A$ ). No critério $B$, o indivíduo precisa colocar em prática esses impulsos sexuais com uma pessoa que não consentiu ou é incapaz de dar consentimento, ou os impulsos/fantasias sexuais devem causar sofrimento clinicamente significativo ou prejuízo no funcionamento social, profissional ou em outras áreas importantes da vida desse indivíduo. Portanto, somente se ambos os critérios (A e B) forem preenchidos é confirmado o diagnóstico de TP. Se somente o critério A ocorrer, não se trata de TP, mas de parafilia. No DSM-5, os TPs são classificados em: transtorno voyeurista, transtorno exibicionista, transtorno frotteurista, transtorno do masoquismo sexual, transtorno do sadismo sexual, transtorno pedofílico, transtorno fetichista, transtorno transvéstico, outros transtornos parafílicos especificados e transtornos parafílicos não especificados (APA, 2013).

Um autor afirmou que são nomeadas pelo menos 40 parafilias, mas não se sabe quantas realmente existem (SHARMA, 2003). Estão descritas parafilias por amputados, idosos e muitas outras (KAUL et al., 1991; KOLLA et al., 2009). Uma publicação listou 549 delas (AGGRAWAL, 2009).

A prevalência de TP é desconhecida, havendo apenas estimativas (APA, 2013; VON FRANQUÉ et al., 2013; OLIVEIRA JÚNIOR, 2015). Muitas são ilegais ou causam constrangimento. $O$ indivíduo, então, tem dificuldades de revelá-las (SHARMA, 2003).

Uma pesquisa mostrou que quase metade dos entrevistados expressou interesse em pelo menos uma categoria parafílica, e aproximadamente um terço teve experiência com tal prática pelo menos uma vez (JOYAL et al.; 2017). Segundo Ahlers et al. (2011), as fantasias parafílicas têm maior probabilidade de existir em solteiros e em pessoas que se masturbam, pelo menos uma vez por semana, e a relação entre ter fantasias pedofílicas e realizá-las na vida real é de $9,5 \%$ e $3,8 \%$, respectivamente.

As possíveis etiologias dos TPs não são bem conhecidas, porém várias hipóteses têm sido formuladas (ABDO, 2012). Etiologias psicológicas, ambientais, orgânicas e de características físicas do indivíduo foram estudadas (CANTOR et al., 2007; DYSHNIKU et al., 2015; FAZIO et al., 2017).

\section{Metodologia}

Foi realizada revisão de literatura no Pubmed e Medline utilizando-se os termos:

- Paraphilias OU PD E treatment;

- Paraphilias OU PD E comorbities;

- Paraphilias OU PD OU sexual dysfunctions E serotonin reuptake inhibitors OU antidepressive agents OU cyproterone OU medroxyprogesterone OU gonadotropin-releasing hormone/agonists OU leuprolide OU triptorelin OU Estrogens OU diethylstilbestrol OU antipsychotic agents OU flutamide OU naltrexone $\mathrm{OU}$ narcotic antagonists $\mathrm{OU}$ androgen antagonists $\mathrm{OU}$ libido/drug effects); (treatment $\mathrm{E}$ voyeurism OU exhibitionism OU frotteurism OU sexual masochism OU sexual sadism OU pedophilia, fetishism E transvestism;

- Paraphilias drug treatment OU PD drug treatment $E$ ethics $O U$ human rights OU law enforcement OU public opinion OU public health OU erotica/legislation E jurisprudence.

\section{Medicamentos e efeitos colaterais}

As medicações usadas para tratar os TPs visam inibir a função sexual e, assim, coibir o comportamento parafílico. Os principais mecanismos para que tal objetivo seja alcançado são a inibição dopaminérgica e da testosterona, além da ativação serotoninérgica. Podem ser usados antidepressivos, antiandrógenos e outros medicamentos, dependendo das comorbidades e da gravidade dos TPs, considerando que pacientes resistentes a uma única medicação podem se beneficiar de uma associação de várias (BRIKEN et al., 2013). Assim, as principais drogas descritas na literatura (cujos efeitos colaterais são apresentados no Quadro 1) são:

- Antidepressivos (ADs): podem diminuir o 
comportamento parafílico; em geral, não impedem que uma relação sexual seja realizada com prazer. São utilizados em casos de transtornos menos graves. Tem efeito sobre a sexualidade, principalmente pela ação da serotonina no receptor pós-sináptico 5HT2a. Esse efeito inibe a ação dopaminérgica nos centros mesolímbicos do prazer. Podem inibir o óxido nítrico sintetase, prolongar o orgasmo por inibição do reflexo medular da ejaculação, provocar anestesia genital e aumentar a prolactina. Os tricíclicos inibem a sexualidade também por ação anticolinérgica (inibindo a inervação parassimpática da ereção). Por meio desses mecanismos, portanto, o desejo, a excitação e o orgasmo podem ser prejudicados (HALLWARD et al., 2001; STAHL, 2013). Muitos parafílicos apresentam depressão e ansiedade como comorbidades, sendo que as próprias fantasias e os atos parafílicos podem gerar culpa e depressão. Drogas serotoninérgicas como os inibidores seletivos de recaptação de serotonina (ISRS) podem mediar a natureza obsessivo-compulsiva das fantasias sexuais, inibindo a frequência e a intensidade dessas fantasias, além de tratar a depressão e a ansiedade. Assim, os ADs diminuem o comportamento gerado por esse apetite sexual exacerbado e trata as comorbidades (ABDO, 2012; ASSUMPÇÃO et al., 2014). Por outro lado, pacientes que fazem uso de antiandrógenos podem se tornar depressivos e, portanto, os ADs podem ser usados para tratar também esse efeito colateral daquelas medicações (LEE et al., 2015).

- Antiandrógenos: (Ciproterona, Medroxiprogesterona e Agonistas LHRH):

- Ciproterona (CPA): preparado hormonal de efeito antiandrogênico que inibe a resposta sexual de maneira mais intensa que os antidepressivos, com redução particularmente das fantasias sexuais e dos níveis dos hormônios sexuais. Faz inibição competitiva dos receptores de androgênios das células-alvo. Também possui ação progestágena que resulta na inibição de produção de gonadotrofinas pela hipófise. Reduz os níveis plasmáticos de testosterona, LH e FSH. A apresentação oral de uso diário dificulta a adesão, que melhora com a aplicação intramuscular semanal (BRADFORD; PAWLAK, 1993; CODISPOTI, 2008; GUAY, 2009; MIMURA, 2015).

- Medroxiprogesterona (MPA): inibe a resposta sexual em intensidade semelhante à ciproterona (COOPER et al., 1992). É um derivado da progesterona e age inibindo o FSH e o $\mathrm{LH}$, diminuindo os níveis sanguíneos de ACTH e hidrocortisona, reduzindo a testosterona circulante por meio da inibição da 5 a redutase hepática e diminuindo o estrógeno (no caso de mulheres). É administrada via intramuscular a cada três meses, o que facilita a adesão (GUAY, 2009; BUMERAD, 2014; THIBAUT et al., 2016).

- Agonistas parciais do hormônio liberador de gonadotrofina hipotalâmico (agonistas LHRH): causam maior inibição do comportamento parafílico do que a CPA e a MPA (BRIKEN et al., 2003), podendo levar a testosterona a níveis de castração. São análogos sintéticos do LHRH hipotalâmico, sendo que a administração crônica dessas drogas provoca a inibição da produção do LH pela hipófise. Assim, inibem a testosterona no homem e o estradiol na mulher. $\mathrm{O}$ uso de agonistas LHRH é indicado, portanto, para quem oferece perigo a outrem. Por outro lado, essas drogas levam à maior inibição no desejo do que na ereção. Assim, apesar de mais improvável, a ereção pode acontecer. Mesmo a castração física (orquidectomia) não impede a ereção. Seus principais representantes são a gosserrelina, o leuprolide e a triptorrelina. As aplicações por meio de injeções mensais ou trimestrais facilitam a adesão (BRIKEN et al., 2003; GARCIA et al., 2011; TEIXEIRA, 2015; THIBAUT et al., 2016).

- Flutamida: é um antiandrogênico, usada em comprimidos três vezes ao dia. É hepatotóxica, necessitando de monitorização hepática constante (ROUSSEAU; COUTURE; DUPONT; LABRIE; COUTU$\mathrm{RE}, 1990)$.

Quanto aos efeitos colaterais dos antiandrógenos (Quadro 1), a osteoporose, hepatotoxicidade, depressão e infertilidade podem ocorrer nos agonistas LHRH, na MPA e CPA. Uma especial atenção deve ser direcionada à hepatotoxicidade da ciproterona, às lesões vasculares retinianas, ao ganho de peso da MPA e à osteoporose dos agonista LHRH (BUMERAD, 2014; MIMURA, 2015; TEIXEIRA, 2015).

- Estrógenos: são medicações de segunda ou terceira linha para TP. A necessidade de uso diário de comprimidos dificulta a adesão (GUAY; 2009).

- Antipsicóticos (APs): agem, principalmente pelo efeito antidopaminérgico; e, também por este mecanismo, inibem a função sexual. São indicados principalmente para pacientes psicóticos e bipolares (estes, mesmo se não psicóticos). Injeções quinzenais ou mensais facilitam a adesão (STAHL, 2013; KHAN et al., 2015).

- Naltrexona: é um antagonista opiáceo com ação antidopaminérgica e que pode auxiliar no tratamento dos pacientes com comportamento sexual compulsivo (parafílico ou não). Assim, tratam etilismo e TP associados. É contraindicado se associado a opioides e na sua síndrome de abstinência 
(RYBACK, 2004; MÓDOLO, 2014).

- Estabilizadores do humor (EHs): são usados nos pacientes bipolares e impulsivos (STAHL, 2013), podendo também inibir a sexualidade. Acredita-se que os anticonvulsivantes inibem a função sexual pelo efeito gabaérgico. São eles: lítio (KAFKA,
1991) e anticonvulsivantes como ácido valproico (WANG et al., 2014), carbamazepina (VARELA et al., 2002); oxcarbazepina (CORRETTI et al., 2010) e topiramato (SHIAH et al., 2006).

Quadro I - Efeitos colaterais dos medicamentos usados para TP (STAHL, 2013; BUMERAD, 2014; MÓDOLO, 2014; MIMURA, 2015; TEIXEIRA, 2015)

Medicamentos

AD ISRS

AD Tricíclicos

Ciproterona

Medroxiprogesterona

Agonistas LHRH

Estrógenos

Antipsicóticos

Naltrexona

Flutamida

\section{Efeitos colaterais das medicações (além das disfunções sexuais)}

Aumento de peso, alterações gastrointestinais, tremores, sonolência, insônia, entre outros.

Mesmos dos AD ISRS e: hipotensão postural, boca seca, inibição da condução intracardíaca, convulsões, entre outros. Esses últimos efeitos são mais frequentes com os tricíclicos do que com o AD ISRS.

Hepatotoxicidade, depressão, anemia hipocrômica, ginecomastia, alterações de PA e do eletrocardiograma, infarto do miocárdio, lesões tromboembólicas, letargia, cefaleia, anormalidades lipídicas, ganho de peso, insuficiência cardíaca, hipoespermatogênese, fadiga, ondas de calor, sintomas oftalmológicos.

Depressão, lesões retinianas, retenção hídrica, perda óssea, ganho de peso, depressão, alterações da função hepática, icterícia, alterações menstruais, distúrbios gastrointestinais, galactorreia, anovulação, distúrbios tromboembólicos, síndrome de Cushing, pesadelos, ondas de calor, acne, hiperglicemia, diabetes melitus, hipogonadismo, hipoespermogênese, hipertensão, litíase biliar.

Perda da densidade mineral óssea, aumento dos valores de testosterona na primeira semana de tratamento, hiperidrose, edema, depressão, náuseas e vômitos, ondas de calor, dor óssea, redução da tolerância à glicose, reações nos locais das injeções, dificuldade para urinar, alterações de pressão arterial, tumor benigno de hipófise, alterações psicóticas, leucopenia, aplasia de células vermelhas, infarto do miocárdio, arritmias cardíacas, anafilaxia, sangramentos gastrointestinais, atrofia testicular, cefaleia, tontura, acne, apoplexia pituitária (raro).

Indução a neoplasias, doenças biliares e tromboembólicas, depressão, sintomas gastrointestinais, edema periférico, irregularidades menstruais, intolerância à glicose, enxaqueca, coreia.

Impregnações, aumento de peso, síndrome metabólica, hipotensão postural, sonolência, alterações gastrointestinais, alterações de eletrocardiograma, depressão.

Hepatoxicidade, náuseas, cefaleia, depressão.

Hepatotoxicidade, depressão, anemia, sintomas gastrointestinais, ondas de calor. 


\section{Cuidados com antiandrógenos}

Segundo a literatura, caso o TP seja mais grave e com risco de agressão sexual, os antiandrógenos (CPA, MPA e agonistas LHRH) podem ser úteis. Essas medicações diminuem muito a libido, principalmente os agonistas LHRH, tornando a ereção bem mais dificultosa (ROUSSEAU et al., 1990; COOPER et al., 1992; BRADFORD; PAWLAK, 1993; CODISPOTI, 2008; GUAY, 2009; GARCIA et al., 2011; ASSUMPÇÃO et al., 2014; KHAN et al., 2015; HOLOYDA et al., 2016).

Em homens, após cerca de 21 dias da primeira injeção de um agonista LHRH, a testosterona cai para níveis de castração e assim permanece durante o tratamento contínuo. No começo, os agonistas LHRH provocam, no entanto, aumento de testosterona. Assim, pode-se usar CPA, por exemplo, durante as duas primeiras semanas para inibir perifericamente a ação do hormônio masculino (BRIKEN et al., 2003; KRUEGER et al., 2011; HUYGH et al., 2015; TEIXEIRA, 2015). Por outro lado, indivíduos que fazem uso de testosterona exógena para neutralizar o efeito dos agonistas LHRH podem ser detectados na dosagem hormonal. Neste caso, também a associação de CPA ao agonista LHRH pode reverter esse aumento do nível dos hormônios (HUYGH et al., 2015).

Os antiandrógenos podem gerar inúmeros efeitos colaterais, incluindo osteoporose e infertilidade. Antes de iniciar o tratamento, portanto, é necessário avaliar os fatores de risco para os principais efeitos colaterais. É importante realizar bom histórico clínico, exame físico e triagem de exames endócrinos, hematológicos, renais e hepáticos para detectar possíveis problemas pré-existentes, como fatores de risco para osteoporose e infertilidade (BRADFORD; PAWLAK, 1993; GUAY, 2009).

Para evitar a osteoporose, podem ser acrescentados medicamentos como o etidronate, o cálcio, a vitamina $D$ e o ácido zoledrônico, sendo que a CPA e a MPA podem gerar menos rarefação óssea do que os agonistas LHRH (GRASSWICK et al., 2003; KRUEGER et al., 2011; TEIXEIRA, 2015). A ciproterona pode causar hepatite fulminante, por isso é importante monitorar a função hepática (BESSONE et al., 2016). Pode haver atrofia dos túbulos seminíferos e das células de Leyding, gerando infertilidade (GARCIA et al.; 2011; KRUEGER et al., 2011; TEIXEIRA, 2015).

Esses medicamentos são contraindicados para menores de dezoito anos, em indivíduos com maturação óssea ou testicular incompleta, com patologias hipofisárias e hipersensibilidade aos componentes das drogas (TEIXEIRA, 2015). Os antiandrógenos devem ser evitados em portadores de tumores malignos (exceto câncer de próstata), doenças hepáticas, tromboembolismo, diabetes severa com alterações vasculares, depressão grave crônica, anemia, doenças debilitantes e osteoporose prévias, bem como doenças pituitárias ativas; exames precisam ser monitorados periodicamente (GRASSWICK et al., 2003; TEIXEIRA, 2015). Como os TPs são doenças crônicas, é recomendado que o tratamento seja longo, de pelo menos três a cinco anos, principalmente para TP com risco de agressividade ou violência sexual (GARCIA et al., 2011). Não se sabe ainda todas as reações e consequências em longo prazo do uso dos agonistas LHRH, CPA ou MPA. Novos protocolos podem, portanto, vir a ser necessários (KRUEGER et al., 2011; KHAN et al., 2015).

\section{Bioética}

Conforme o(s) diagnóstico(s) do parafílico, o tratamento mais adequado pode variar, sendo necessário saber se há comorbidades (JAIRAM et al., 2008), qual o tipo de TP e sua gravidade; enfatizando, principalmente, o risco de agressão sexual. Outros diagnósticos psiquiátricos ou clínicos concomitantes precisam ser, portanto, considerados (BRIKEN al., 2003).

Um indivíduo depressivo que tem fantasias pedofílicas e que nunca realizou nenhum ato real é muito diferente de um sociopata que estupra e mata crianças. O primeiro indivíduo pode ser considerado portador de transtorno pedofílico porque tem sofrimento, preenchendo o critério B do DSM5 (APA, 2013). Neste caso, o uso de um ISRS pode aliviar os sintomas da depressão e diminuir a libido pelo efeito serotoninérgico (STAHL, 2013).

Um oligofrênico que faz carícias íntimas numa criança é muito diferente de indivíduos sem esse quadro que realizam o mesmo ato, tornando as abordagens psicoterápicas e medicamentosas muito importantes (KEELING et al., 2005; REA et al., 2017). Um esquizofrênico que também é agressor sexual precisa usar antipsicóticos, que diminuem a libido pelo efeito antidopaminérgico (GARCIA et al., 2011; STAHL, 2013).

Fantasiar relações sexuais com animais é muito comum, principalmente em indivíduos criados no meio rural (OLIVEIRA JÚNIOR; ABDO, 2010). Concretizar essas fantasias, porém, pode ser considerado crime em alguns países (HVOZDIK et al., 2006). Uma classificação para a zoofilia a subdividiu em dez tipos, indo desde o zoófilo romântico, pas- 
sando pelo zoófilo fantasioso até o zoófilo sádico, que tortura e mata o animal. O necrófilo tem uma classificação semelhante e o mesmo poderia ser feito para outras parafilias (AGGRAWAL, 2011).

Para o sadismo e o masoquismo consensuais já existem discussões de leis específicas em alguns países. Os rituais sadomasoquistas podem variar desde níveis bem leves até casos de hipoxifila, em que um praticante pode morrer asfixiado mesmo que tenha consentido no ato (RIDINGER, 2006; WHITE, 2006; WRIGHT, 2006).

Um indivíduo que passa a praticar atos sexuais com crianças após desenvolver um tumor cerebral é muito diferente de outro que não tenha tal patologia (GILBERT et al., 2015). No entanto, é importante que a sociedade seja protegida de agressores sexuais, sejam eles parafílicos (no caso dos pedófilos, necrófilos, exibicionistas, voyeures, frotteuristas), ou não parafílicos (no caso dos estupradores de adultos); sendo fundamental o diagnóstico quanto à possiblidade de agressão (LEWIS et al., 2017).

Esta avaliação de risco não é uma tarefa fácil. Até as pesquisas com TP encontram barreiras éticas. Recentemente, por exemplo, um estudo sobre o uso de Triptorrelina nos TPs foi impedido de ser realizado na Alemanha (BRIKEN et al., 2017). Escalas de avaliação de risco de recidivas foram, então, desenvolvidas com a intenção de tornar mais objetivo o trabalho dos profissionais (LOOMAN et al., 2013; SETO et al., 2015; RASMUSSEN, 2017; REEVES et al., 2017).

Quanto ao tratamento medicamentoso, a literatura indica, de modo geral, os IRSSs para TP de média gravidade e antiandrógenos (MPA, CPA e agonistas LHRH) em casos mais graves, com risco de agressão sexual (MELELLA et al., 1989; BRIKEN et al., 2003, 2017; CODISPOTI, 2008).

Os antiandrógenos diminuem acentuadamente a libido e geram muitos efeitos colaterais, podendo o indivíduo usá-los de forma voluntária ou involuntária, por ordem judicial. Em ambos os casos, o indivíduo deve assinar um documento de que está ciente de tudo que pode acontecer devido ao uso dessas drogas e de que deve monitorar periodicamente os exames acima descritos. Assim, o uso dessas medicações pode ser uma alternativa à prisão (MELELLA et al., 1989; LE DARE et al., 2015; TURNER et al., 2017). Por outro lado, a orquidectomia é um recurso extremo que diminui muito a libido, mas não impede as ereções. Porém, ao contrário do uso de antiandrógenos, é uma castração definitiva (LEE et al., 2013; ASSUMPÇÃO et al, 2014). Um estudo mostrou que estimular a região ventromedial do hipotálamo pode diminuir a libido (FUSS et al., 2015).

Assim, diante de tal complexidade terapêutica, é prudente que as drogas (antidepressivos, antiandrógenos ou outras) sejam prescritas por médicos experientes no uso de cada uma delas (SALEH et al., 2010; GARCIA et al., 2011; SILVANI et al., 2015). Salientando-se, no entanto, que os antiandrógenos não são permitidos no Brasil para tratamento de TP. Em relação às escalas de avaliação de risco, elas também só devem ser avaliadas por profissionais experientes em sua utilização (LOOMAN et al., 2015; RASMUSSEN, 2017; REEVES et al., 2017).

Caso um pedófilo mencione para seu terapeuta que esteja realizando um ato real de pedofilia, o profissional deve denunciar à justiça a fim de que a criança seja protegida. Cada caso deve ser discutido entre psiquiatras e juristas antes que se tome uma decisão de castração química, pois o assunto é muito delicado (SCOTT et al., 2003; GUAY, 2009; SALEH et al., 2010). Uma mulher (por exemplo) pode acusar de pedofilia o marido que a traiu só para vê-lo castrado quimicamente. O ideal, no entanto, é o desenvolvimento de drogas eficazes e com menos efeitos colaterais (GUAY, 2009; ASSUMPÇÃO et al, 2014). Os direitos humanos de todos (vítima, agressor sexual e público em geral) devem ser preservados (LE DARE et al., 2015; THIBAUT et al., 2016).

\section{Considerações finais}

Segundo o DSM-5, parafilias não são doenças e, portanto, não devem ser tratadas. Porém, quando se trata de TP, o assunto é polêmico e muito delicado. O melhor medicamento indicado para cada caso depende do diagnóstico do TP, das comorbidades psiquiátricas/clínicas, da gravidade da parafilia, dos efeitos terapêuticos e do risco de efeitos colaterais (VOLPE et al., 2000; BRIKEN et al., 2003). Volpe e Tavares (2000) descreveram o caso de um paciente com doença de Wilson (DW) que desenvolveu um surto psicótico diagnosticado como orgânico e acompanhado de exibicionismo. O paciente foi tratado da DW com d-penicilamida, que depois foi substituída por citrato de zinco, e não houve alteração da excreção urinária de cobre. Usou, então, neurolépticos que reverteram o quadro psicótico. Porém, o exibicionismo persistiu (apesar do efeito antidopaminérgico do neuroléptico), sendo adicionada ciproterona, que reverteu o TP.

Em linhas gerais, os TPs de menor gravidade podem ser tratados com antidepressivos e os de maior gravidade com antiandrógenos (BRIKEN 
et al., 2003; CODISPOTI, 2008). Dependendo das comorbidades, outras medicações que também diminuem a libido podem ser importantes, como é o caso de antipsicóticos nos pacientes psicóticos (STAHL, 2013; KHAN et al., 2015), Estabilizadores de humor nos bipolares (KAFKA, 1991; VARELA et al., 2002; SHIAH et al., 2006; CORRETTI et al., 2010; WANG et al., 2014) e naltrexona nos etilistas (RYBACK, 2004; MÓDOLO, 2014). Essas drogas, juntamente com a psicoterapia, são de grande valia na prevenção de recidivas (LASHER et al., 2017).

Muitos portadores de TP não realizam o ato parafílico, mas têm sofrimento pelas suas fantasias, como depressão e disfunções sexuais. O paciente pode necessitar de intervenção medicamentosa e psicoterapêutica visando, à medida do possível, que seja tratado com preservação de sua vida sexual. Para ofensores sexuais, no entanto, a inibição da libido pode ser necessária. O benefício das medicações deve superar o risco dos efeitos colaterais, e a colaboração do indivíduo com o tratamento é desejável. Muitos só se tratam, no entanto, por ordem judicial (LE DARE et al., 2015; TURNER et al., 2017).

Reforçando que no Brasil o uso de antiandrógenos para o tratamento de TP não é liberado.

\section{Referências}

ABDO CHN. Sexualidade humana e seus transtornos. $4^{\mathrm{a}}$ ed. São Paulo: Casa Leitura Médica, 2012.

AGGRAWAL A. A new classification of zoophilia. Journal of Forensic and Legal Medicine, v. 18, n. 2, p. 73-78, fev. 2011.

AGGRAWAL A. Forensic and medico-legal aspects of sexual crimes and unusual sexual practices. Boca Raton: CRC Press, 2009.

AHLERS, C. J.; SCHAEFER, G. A.; MUNDT, I. A.; ROLL, S.; ENGLERT, H.; WILLICH, S. N.; BEIER, K. M. How unusual are the contents of paraphilias? Paraphilia-associated sexual arousal patterns in a community-based sample of men. Journal of Sexual Medicine, v. 8, n. 5, p. 1362-1370, maio 2011.

AMERICAN PSYCHIATRIC ASSOCIATION (APA). Diagnostic and statistical manual of mental disorders-DSM-5. Arlignton: American Psychiatric Association, 2013.

ASSUMPÇÃO, A. A.; GARCIA, F. D.; GARCIA, H. D.; BRADFORD, J. M.; THIBAUT, F. Pharmacologic treatment of paraphilias. Psychiatric clinics of North
America, v. 37, n. 2, p. 173-181, jun. 2014.

BESSONE, F.; LUCENA, M. I.; ROMA, M. G.; STEPHENS, C.; MEDINA-CÁLIZ, I.; FRIDER, B.; TSARIKTSIAN, G.; HERNÁNDEZ, N.; BRUGUERA, M.; GUALANO, G.; FASSIO, E.; MONTERO, J.; REGGIARDO, M. V.; FERRETTI, S.; COLOMBATO, L.; TANNO, F.; FERRER, J.; ZENO, L.; TANNO, H.; ANDRADE, R. J. Cyproterone acetate induces a wide spectrum of acute liver damage including corticosteroid-responsive hepatitis: report of 22 cases. Liver International, v. 36, n. 2, p. 302-310, fev. 2016.

BRADFORD, J. M.; PAWLAK, A. Double-blind placebo cross over study of Cyproterona Acetate in the treatment of the paraphilias. Archives of Sexual Behavior, v. 22, n. 5, p. 383-402, out. 1993.

BRIKEN, P.; HILL, A.; BERNER, W. Pharmacotherapy of paraphilias with long-acting agonists of luteinizing hormone-releasing hormone: a systematic review. Journal of Clinical Psychiatry, v. 64, n. 8, p. 890-897, ago. 2003.

BRIKEN, P.; MÜLLER, J. L.; BERNER, W.; BÖDEKER, R. H.; VOLLMANN, J.; KASPERK, C.; KOLLER, M. Failure of a study in forensic psychiatric hospitals: clinical trial to investigate the additive effect of triptorelin on the efficacy of psychotherapy. Nervenarzt, v. 88, n. 5, p. 480-485, maio 2017.

BUMERAD, J. C. Depo-provera: acetato de medroxiprogesterona [bula de remédio]. Guarulhos: Pfizer, 2014.

CANTOR, J. M.; KUBAN, M. E.; BLAK, T.; KLASSEN, P. E.; DICKEY, R.; BLANCHARD, R. Physical height in pedophilic and hebephilic sexual offenders. Sexual Abuse, v. 19, n. 4, p. 395-407, dez. 2007.

CODISPOTI, V. L. Pharmachology of sexually compulsive behavior. Psychiatric Clinics of North America, v. 31, n. 4, p. 671-679, dez. 2008.

COOPER, A. J.; SANDHU, S.; LOSZTYN, S.; CERNOVSKY, Z. A double-blind placebo controlled trial of medroxyprogesterone acetate and cyproterone acetate with seven pedophiles. Canadian Journal of Psychiatry, v. 37, n. 10, p. 687-693, dez. 1992.

CORRETTI, G.; BALDI, I. Oxcarbazepine reduces exhibitionist urges and behaviors in a paraphilic patient. Archives of Sexual Behavior, v. 39, n. 5, p. 10251026, out. 2010. 
De BLOCK, A.; ADRIAENS, P. R. Pathologizing sexual deviance: a history. Journal of Sex Research, v. 50, n. 3-4, p. 276-298, 2013.

DOUGLAS, T.; BONTE, P.; FOCQUAERT, F.; DEVOLDER, K.; STERCKX, S. Coercion, incarceration, and chemical castration: an argument from autonomy. Journal of Bioethical Inquiry, v. 10, n. 3, p. 393-405, out. 2013.

DYSHNIKU, F.; MURRAY, M. E.; FAZIO, R. L.; LYKINS, A. D.; CANTOR, J. M. Minor physical anomalies as a window into the prenatal origins of pedophilia. Archives of Sexual Behavior, v. 44, n. 8, p. 2151-2159, nov. 2015.

FAZIO, R. L.; DYSHNIKU, F.; LYKINS, A. D.; CANTOR, J. M. Leg length versus torso length in pedophilia: further evidence of atypical physical development early in life. Sexual Abuse, v. 29, n. 5, p. 500-514, ago. 2017.

FEDOROFF, J. P. Forensic and diagnostic concerns arising from the proposed DSM- 5 criteria for sexual paraphilic disorder. Journal of the American Academy of Psychiatry and the Law, v. 39, n. 2, p. 238241, 2011.

FEDOROFF, J. P.; DI GIOACCHINO, L.; MURPHY, L. Problems with paraphilias in the DSM-5. Current Psychiatry Reports, v. 15, n. 8, p. 363, ago. 2013.

FUSS, J.; AUER, M. K.; BIEDERMANN, S. V.; BRIKEN, P.; HACKE, W. Deep brain stimulation to reduce sexual drive. Journal of Psychiatry \& Neuroscience, v. 40, n. 6, p. 429-431, nov. 2015.

GARCIA, F. D.; THIBAUT, F. Current concepts in the pharmacotherapy of paraphilias. Drugs, v. 71, n. 6, p. 771-790, abr. 2011.

GILBERT, F.; FOCQUAERT, F. Rethinking responsibility in offenders with acquired paedophilia: punishment or treatment? International Journal of Law and Psychiatry, v. 38, p. 51-60, jan./fev. 2015.

GRASSWICK, L. J.; BRADFORD, J.M. Osteoporosis associated with treatment of paraphilia: a clinical review of seven cases reports. Journal of Forensic Science, v. 48, n. 4, p. 849-855, jul. 2003.

GUAY, D. R. Drug treatment of paraphilic and non paraphilic sexual disorders. Clinical Therapeutics, v. 31, n. 1, p. 1-31, jan. 2009.
HALLWARD, A.; ELLISON, J. M. Antidepressants and sexual function. Londres: Harcourt Health Communications, 2001.

HOLOYDA, B. J.; KELLAHER, D. C. The biological treatment of paraphilic disorders: an updated review. Current Psychiatry Reports, v. 18, n. 2, p. 19, fev. 2016.

HUYGH, J.; VERHAEGEN, A.; GOETHALS, K.; COSYNS, P.; De BLOCK, C.; van GAAL, L. Prolonged flare-up of testosterone after administration of a gonadotrophin agonist to a sex offender: an under-recognised risk? Criminal Behaviour and Mental Health, v. 25, n. 3, p. 226-230, jul. 2015.

HVOZDIK, A.; BUGARSKÝ, A.; KOTTFEROVÁ, J.; VARGOVÁ, M.; ONDRASOVICAVÁ, O.; ONDRASOVIC, M.; SASÁKOVÁ, N. Ethological, psychological and legal aspects of animal sexual abuse. Veterinary Journal, v. 172, n. 2, p. 374-376, set. 2006.

JAIRAM, J.; van MARLE, $H$. J. The treatment of hypersexuality in a male with obsessive compulsive disorder as psychiatric co-morbidity. TijdschrVoorPsychiatrie, v. 50, n. 2, p. 113-117, 2008.

JOYAL, C. C.; CARPENTIER, J. The prevalence of paraphilic interests and behaviors in the general population: a provincial survey. Journal of Sex Research, v. 54, n. 2, p. 161-171, fev. 2017.

KAFKA, M. P. Successful antidepressant treatment of nonparaphilic sexual addictions and paraphilias in men. Journal of Clinical Psychiatry, v. 52, n. 2, p. 60-65, fev. 1991.

KAPFHAMMER, H. P. Richard Freiherr v. Krafft-Ebing and Sigmund Freud - discourse on the "normality" and "perversion" of human sexuality at the close of the 19th century and the beginning of the 20th century. Neuropsychiatrie, v. 29, n. 4, p. 163-178, 2015.

KAUL, A.; DUFFY, S. Gerontophilia: a case report. Medicine, Science, and the Law, v. 31, n. 2, p. 110114, 1991.

KEELING, J. A.; ROSE, J. L. Relapse prevention with intellectually disabled sexual offenders. Sexual $\mathrm{Abu}$ se, v. 17, n. 4, p. 407-423, out. 2005.

KHAN, O.; FERRITER, M.; HUBAND, N.; POWNEY, M. J.; DENNIS, J. A.; DUGGAN, C. Pharmacological in- 
terventions for those who have sexually offended or are at risk of offending. Cochrane Database Systematic Reviews, n. 2, p. CD007989, fev. 2015.

KOLLA, N. J.; ZUCKER, K. J. Desire for non-mutilative disability in a nonhomosexual, male-to-female transsexual. Archives of Sexual Behavior, v. 38, n. 6, p. 1057-1063, 2009.

KRAFFT-EBING, R. Psychopathiasexualis. the classic study of deviant sex. New York: Arcade Publishing, 1965.

KRUEGER, R. B. The DSM diagnostic criteria for sexual sadism. Archives of Sexual Behavior, v. 39, n. 2, p. 325-345, abr. 2010a.

KRUEGER, R. B. The DSM diagnostic criteria for sexual masochism. Archives of Sexual Behavior, v. 39, n. 2, p. 346-356, abr. 2010b.

KRUEGER, R. B. Critical appraisals of the proposed DSM-5 paraphilia diagnoses. Journal of the American Academy of Psychiatry and the Law, v. 39, n. 2, p. 237, 2011.

KRUEGER, R.B.; KAPLAN, M.S. Depot-leuprolide for treatment of paraphilias: a report of twelve cases. Archives of Sexual Behavior, v. 30, n. 4, p. 409-422, ago. 2011.

LASHER, M. P.; STINSON, J. D. Adults with pedophilic interests in the United States: current practices and suggestions for future policy and research. Archives of Sexual Behavior, v. 46, n. 3, p. 659-670, abr. 2017.

LE DARE, B.; JEHANNIN, A.; LANOE, F.; PHILIPPE, F.; TASSEL, C.; ABONDO, M.; MARIE, N. Treatment management of sexual offenders. Annales Pharmaceutiques Françaises, v. 73, n. 4, p. 257-265, jul. 2015.

LEE, J. Y.; CHO, K. S. Chemical castration for sexual offenders: physicians' views. Journal of Korean Medical Science, v. 28, n. 2, p. 171-172, fev. 2013.

LEE, M.; JIM, H. S.; FISHMAN, M.; ZACHARIAH, B.; HEYSEK, R.; BIAGIOLI, M.; JACOBSEN, P. B. Depressive symptomatology in men receiving androgen deprivation therapy for prostate cancer: a controlled comparison. Psychooncology, v. 24, n. 4, p. 472477, abr. 2015.

LEWIS, A.; GRUBIN, D.; ROSS, C. C.; DAS, M. Go- nadotrophin-releasing hormone agonist treatment for sexual offenders: a systematic review. Journal of Psychopharmacology, v. 31, n. 10, p. 1281-1293, out. 2017.

LOOMAN, J.; MORPHETT, N. A.; ABRACEN, J. Does consideration of psychopathy and sexual deviance add to the predictive validity of the static-99R? International Journal of Offender Therapy and Comparative Criminology, v. 57, n. 8, p. 939-965, ago. 2013.

MELELLA, J. T.; TRAVIN, S.; CULLEN, K. Legal and ethical issues in the use of antiandrogens in treating sex offenders. Bulletin of the American Academy of Psychiatry and the Law, v. 17, n. 3, p. 223-232, 1989.

MIMURA, D. E. Androcur. acetato de ciproterona [bula de remédio]. São Paulo: Bayer, 2015.

MÓDOLO, J. C. Revia: cloridrato de naltrexona [bula de remédio]. Itapira: Cristália, 2014.

MONEY, J. History, causality, and sexology. Journal of Sex Research, v. 40, n. 3, p. 237-239, 2003.

OLIVEIRA JÚNIOR, W. M.; ABDO, C. H. Unconventional sexual behaviors and their associations with physical mental and sexual health parameters: a study in 18 large Brazilian cities. Revista Brasileira de Psiquiatria, v. 32, n. 3, p. 264-274, set. 2010.

OLIVEIRA JÚNIOR, W. M. Impact of psychotherapy on the choice of sexual partners with paraphilia on drug treatment. Tese (doutorado) - Faculdade de Medicina, Universidade de São Paulo, São Paulo, 2015.

RASMUSSEN, L. A. L. Comparing predictive validity of JSORRAT-II and MEGA with sexually abusive youth in long-term residential custody. International Journal of Offender Therapy and Comparative Criminology, set. 2017 [No prelo].

REA, J. A.; DIXON, M. R.; ZETTLE, R. D.; WRIGHT, K. $\mathrm{L}$. The development of in vivo measures to assess the impact of sex-drive reducing medications in an offender with an intellectual disability. Archives of Sexual Behavior, v. 46, n. 3, p. 843-859, abr. 2017.

REEVES, S. G.; OGLOFF, J. R. P.; SIMMONS, M. The predictive validity of the Static-99, Static-99R, and Static-2002/R: which one to use? Sexual Abuse, jun. 
2017. [No prelo].

RIDINGER, R. B. Negotiation limits: the legal status of $\mathrm{s} / \mathrm{m}$ in the United States. Journal of Homossexuality, v. 50, n. 2-3, p. 189-216, 2006.

ROUSSEAU, L.; COUTURE, M.; DUPONT, A.; LABRIE, F.; COUTURE, N. Effect of combined androgen blockade with an LHRH agonist and flutamide in one severe case of male exhibitionism. Canadian Journal of Psychiatry, v. 35, n. 4, p. 338-341, maio 1990.

RYBACK, R. S. Naltrexone in the treatment of adolescent sexual offenders. Journal of Clinical Psychiatry, v. 65, n. 7, p. 982-986, Jul 2004.

SALEH, F. M.; GRUDZINSKAS-JR, A. J.; MALIN, H. M.; DWYER, R. G. The management of sex offenders: perspectives for psychiatry. Harvard Review of Psychiatry, v. 18, n. 6, p. 359-368, nov./dez. 2010.

SCOTT, C. L.; HOLMBERG, T. Castration of sex offendersprisoners rights versus public safety. Journal of the American Academy of Psychiatry and the Law, v. 31, n. 4, p. 502-509, 2003.

SETO, M. C.; EKE, A. W. Predicting recidivism among adult male child pornography offenders: development of the Child Pornography Offender Risk Tool (CPORT). Law and Human Behavior, v. 39, n. 4, p. 416-429, ago. 2015.

SHARMA, B. R. Disorders of sexual preference and medicolegal issues thereof. American Journal of Forensic Medicine and Pathology, v. 24, n. 3, p. 277282, set. 2003.

SHIAH, I. S.; CHAO, C. Y.; MAO, W. C.; CHUANG, Y. J. Treatment of paraphilic sexual disorder: the use of topiramate in fetishism. International Clinical Psychopharmacology, v. 21, n. 4, p. 241-243, jul. 2006.

SILVANI, M.; MONDAINI, N.; ZUCCHI, A. Androgen deprivation therapy (castration therapy) and pedophilia: what's new. Archivio Italiano di Urologia, Andrologia, v. 87, n. 3, p. 222-226, set. 2015.

STAHL, S. M. Stahl's essential psychopharmacology. neuroscientific basis and practical applications. $4^{\text {a }}$ ed. Nova York: Cambridge University Press, 2013.

TEIXEIRA, G. H. V. C. Zoladex. acetato de gosserrelina [bula de remédio]. Cotia: Astra Zenica, 2015.
THIBAUT, F.; BRADFORD, J. M.; BRIKEN, P.; DE LA BARRA, F.; HÄßLER, F.; COSYNS, P.; WFSBP TASK FORCE ON SEXUAL DISORDERS. The World Federation of Societies of Biological Psychiatry (WFSBP) guidelines for the treatment of adolescent sexual offenders with paraphilic disorders. World Journal of Biological Psychiatry, v. 17, n. 1, p. 2-38, 2016.

TURNER, D.; PETERMANN, J.; HARRISON, K.; KRUEGER, R.; BRIKEN, P. Pharmacological treatment of patients with paraphilic disorders and risk of sexual offending: An international perspective. World Journal of Biological Psychiatry, p. 1-10, nov. 2017. [No prelo].

VARELA, D.; BLACK, D. W. Pedophilia treated with carbamazepine and clonazepam. American Journal of Psychiatry, v. 159, n. 7, p. 1245-1246, jul. 2002.

VOLPE, F. M.; TAVARES, A. Cyproterone for hipersexuality in a psychotic patient with Wilson's desease. Australian and New Zealand Journal of Psychiatry, v. 34, n. 5, p. 878-879, out. 2000.

Von FRANQUÉ, F.; KLEIN, V.; BRIKEN, P. Paraphilicdisorders and sexual variations. In: ESSM Educational Committee. The EFS and ESSM syllabus of clinical sexology. Amsterdam: Medix, 2013.

WAKEFIELD, J. C. DSM-5 proposed diagnostic criteria for sexual paraphilias: tensions between diagnostic validity and forensic utility. International Journal of Law and Psychiatry, v. 34, n. 3, p. 195-209, maio/ jun 2011.

WANG, S. C.; KAO, Y. C.; LIU, Y. P. Divalproex sodium and quetiapine treatment of a pedophile with bipolar spectrum disorder. Journal of Neuropsychiatry and Clinical Neurosciences, v. 26, n. 3, p. E47-E48, verão 2014.

WHITE, C. The spanner trials and the changing law on sadomasochism in the U.K. Journal of Homossexuality, v. 50, n. 2-3, p. 167-187, 2006.

WRIGHT, S. Discrimination of SM-identified individuals. Journal of Homossexuality, v. 50, n. 2-3, p. 217-231, 2006. 